INTERNATIONAL HIGHER EDUCATION - NUMBER 68 SUMMER 2012 Pages 23-25.

\title{
The College Entrance Examination in China
}

\author{
LIU HAIFENG
}

Liu Haifeng is professor and director of the Institute of Education, at Xiamen University, China. E-mail: liuhf@xmu.edu.cn.

The university entrance examination (also called Gaokao) in China is one of the earliest established systems in the world for the selection of new higher education students, through unified examinations. Each year, between June 7 and 9, millions of students take the exam at the same time. The number of participants in 2008 was the largest in history_10.5 million participants took the examination. In 2011, there were 9.33 million. Taking the entrance examination is the only channel for most students to enter colleges or universities. The examination scores can determine the candidates' chance and determine the quality and prestige of the university that they could attend.

\section{HISTORY}

Since its establishment in 1952, an entrance examination is not only a pivot between institutions of higher education and schools of secondary education but also a key link between higher education institutions and society. Thus, this policy has always been an 
important aspect of education reform in China and a focal point of concern for the entire education circle and the whole society.

The Great Cultural Revolution broke out in 1966. In the cultural and educational circles, abolishing the entrance examination was taken as a breakthrough for the movement and colleges, and universities ceased to enroll new students for a number of years. From 1972 to 1976, the recommendation method was adopted in university recruitment, and only those youths who had practical experiences were entitled to higher education. In student recommendation, priority was given to candidates' performance at work and not to their academic accomplishments.

After an interval of 11 years, the restoration of the entrance examination in 1977 filled tens of thousands of educated youths with exultation. In 1977, when the entrance examination was reintroduced, the examinations and recruitment were conduced by provincial, municipal, or regional governments. In 1978, the model of the national unified examination and locally organized recruitment was restored. More than 30 years after that, a series of reforms have been initiated and key measures among them.

A further direction of reform is to change the present practice of measuring students of different abilities with a unified examination that has the same requirements. One of the schemes being discussed sees a distinction between regular undergraduate colleges and universities or key universities, on the one hand, and junior colleges on the other. 
Applicants to the former type of schools take national unified examinations, which may include contents outside the secondary school syllabuses. Examinations focus more on the measurement of students' ability, while those who apply to the latter type of schools take examinations based entirely on secondary school teaching syllabuses. In addition, there will be more improvements in the format of examinations.

\section{NEW DEVELOPMENT FROM 2010}

In July 2010, Chinese government promulgated the National Medium and Long-Term Educational Reform and Development Plan, in which chapter 12 is about the "examination and enrollment system reform." Among previous educational reform profiles in China, this is the first time that examination and enrollment became an independent chapter. This shows that the government pays high attention to this issue. In that chapter, the government suggests that "National Education Examination Steering Committee should be established to study how to set up the examination reform program and to guide the reform of university entrance examination practice." This decision indicates the great importance of the the examination and enrollment reforms. 


\section{Positive AND Negative ConseQuences}

The entrance examination improves the selection of qualified individuals for universities, to ensure the quality of freshmen. It also promotes the teaching and learning at the secondary and elementary level. Tests provide an opportunity for examinees to wholly depend on themselves, and successes are also under their control. That feeling of control motivates millions of young people to study hard; and thus, the general level of intellectual ability of the whole nation is increased, and education is also revitalized. Promoting equity is the soul of the testing, and "fairness, equality, and transparency" are the central notions of China's testing policy. Testing also promotes hierarchical mobility, to a large extent. It has been a key channel for students in the rural areas to gain residency in urban areas. Ever since Gaokao was resumed in 1977, it made great contribution in the selection of qualified individuals for higher education, and many of those people are playing important roles in every sector of the society. The fast economic growth in the recent 20 years in China is also partly attributable to the resumption and reforms of the entrance examination.

However, this unified national admissions test also results in some negative consequences to elementary and secondary education. High schools focus their attention on college admissions rate. Students are dedicated to either science or liberal arts, while completely ignoring the other field since it will not be tested on the entrance 
examination. Academic pressures are too high, and workload is too heavy for students. Students' physical health is significantly impaired, and there is a constant increase in the proportion of people suffering from myopia. Students are also confined to certain thinking modes, and their individual creativity is largely suppressed. Similar things happen at the school level: Schools become more similar to each other with academic studies, for higher test scores being the sole and whole purpose of teaching and learning. The practice of "teaching to the test" is prevalent for Gaokao: Tested subjects and contents are the focus, while the untested subjects and contents are completely ignored. This has already resulted in narrowed perspectives of students and concentrated efforts in the examinations, with the only purpose of seeking fame and wealth out of the practice. The intended goal of high school education is greatly distorted. The entrance examination system has its advantages and disadvantages. It is still one of the most important and core issues in Chinese education reform.

\section{IMPORTANCE AND FUTURE}

An admissions system that basically depends on the test score, as judging criterion, demonstrates a trend beyond the maneuver of any person. In theory, it is not the optimal selection tool; but in practice, no better substitute is available for competition. If this important decision role is not played by test scores, then power, money, or 
connections would substitute test scores and become the key components in deciding the admissions status. Thus, a unified test seems an effective tool, to ensure fair competition and competition orders. It is a system compatible with the social and cultural contexts of China, certainly needs constant improvements and reforms, but is not suitable to eliminate it.

It has attracted even greater attention when the advantages and disadvantages of this kind of large-scale selective examination have become fully exposed. It should be revealed that the nationally unified examination does cause some negative impacts on education at the elementary and secondary level, but it also should be noted as protecting admissions decisions from the intervention of many other factors, such as connections among people. Despite the negative consequences, which calls for reforms, the elimination of tests is not a right answer for education in China. In general, the examination and enrollment system in China has been existing for 60 years; and although it needs continuous reform, it suits the Chinese situation and will last for a long time.

With no change on Chinese people's perspective and emphasis on education, the system will not receive a decrease in the severity of competition in those university admissions tests, regardless of an increase in the admissions rate. China has been trying to modify the practice of wholly depending on a standardized test. For example, 
universities have sought different kinds of recommendation systems, but all of the options fell short of their goals due to the intervention of connections. Only through the nationally unified standardized testing can the admissions practice be protected from the contamination of connections. The university entrance examination system in China is definitely going to be more diverse; however, considering the society situation and traditional culture in China, it will remain the main channel for university entrance, for a long period. 